\title{
TWO EXAMPLES ON PREIMAGES OF METRIC SPACES
}

\author{
N. NOBLE
}

\begin{abstract}
Examples are given to show that the product of a compact $T_{1}$-space with a metric space need not be a $k$-space, and that the product of a countably compact Hausdorff space with a metric space need not be a quasi- $k$-space. These examples show that the separation assumptions cannot be omitted in the following two known results: Each Hausdorff perfect preimage of a $k$-space is a $k$-space. Each regular quasi-perfect preimage of a $T_{1}$ quasi- $k$ space is a quasi- $k$-space. Some related results, perhaps of independent interest, are also obtained.
\end{abstract}

1. Introduction. A subset $U$ of a space $X$ is $k$-open (resp.quasi-k-open) if $U \cap K$ is relatively open in $K$ for each compact (resp. countably compact) subset $K$ of $X$, and a space in which each $k$-open (resp. quasi- $k$-open) subset is open is called a $k$-space (resp. quasi-k-space). A continuous closed onto map is perfect (resp. quasi-perfect) if the inverse of each point is compact (resp. countably compact). In [2] Arhangel'skii shows that any Hausdorff perfect preimage of a $k$-space is a $k$-space, and Michael observes in [5, Proposition 6.F.2] that essentially the same proof shows that any regular quasi-perfect preimage of a $T_{1}$ quasi- $k$-space is a quasi- $k$-space. In particular, every Hausdorff paracompact $M$-space (= Hausdorff paracompact $p$-space $=$ Hausdorff perfect preimage of a metric space [1]) is a $k$-space, and every regular $M$-space (=quasi-perfect preimage of a metric space [6]) is a quasi- $k$-space. Recently $E$. Michael has asked whether the Hausdorff and regularity assumptions in these results can be omitted. That they cannot is shown by the following two examples.

1.1 Example. There exists a compact $T_{1}$-space $X$ and a metric space $Y$ such that $X \times Y$ is not a $k$-space.

1.2 ExAmple. There exists a countably compact Hausdorff space $X$ and a metric space $Y$ such that $X \times Y$ is not a quasi- $k$-space.

The desired perfect (resp. quasi-perfect) map is, of course, the projection from $X \times Y$ onto $Y$. The constructions of these examples, which will be given in the next two sections, turn upon the fact that compact nonHausdorff spaces need not be locally compact (in the sense that each

Received by the editors March 3, 1971.

AMS 1969 subject classifications. Primary 5425, 5435, 5456; Secondary 5453.

Key words and phrases. $k$-spaces, quasi- $k$-spaces, perfect preimages, quasi-perfect preimages, $M$-spaces, product spaces, countably compact remainders.

(c) American Mathematical Society 1973 
point has a basis of compact neighborhoods), and countably compact nonregular spaces need not be locally countably compact. This suggests, and our next result shows, that it is the nonlocal nature of closed maps which necessitates the separation restrictions in the mapping theorems quoted above. Let us call a map $f: X \rightarrow Y$ locally closed if, for each point $x$ in $X$, each neighborhood $U$ of $x$ contains a neighborhood $V$ such that $\left.f\right|_{V}: V \rightarrow Y$ is a closed map. Recall that a space $Y$ is generated by a cover $\alpha$ (or has the weak topology with respect to $\alpha$ or is an $\alpha$-space) if a subset $U$ of $Y$ is open whenever $U \cap A$ is relatively open in $A$ for each $A$ in $\alpha$. (Thus $k$-spaces are generated by their compact subsets, quasi- $k$-spaces by their countably compact subsets.) The proof of Arhangel'skirı's mentioned above [2, proof of Theorem 2.5] adapts easily to show:

1.3 Theorem. Let $f: X \rightarrow Y$ be continuous and locally closed. If $Y$ is generated by a cover $\alpha$ then $X$ is generated by the cover $f^{-1}(\alpha)$.

Note that to show, for example, that a space is a $k$-space it suffices to show that it is generated by a family of subsets which are themselves $k$-spaces. Thus the theorem of D. E. Cohen [3] which asserts that the product of a locally compact space with a $k$-space is a $k$-space [3] follows from 1.3 and the fact that the projection along a compact space is always closed.

\section{Construction of Example 1.1.}

2.1 Lemma. Let $X_{0}$ be an open subspace of a space $X$, and let $Y$ be $a$ space such that, for each compact subset $K$ of $Y, X_{0} \times K$ is a $k$-space. If $X_{0} \times Y$ is not a $k$-space, then neither is $X \times Y$.

Proof. Let $U \subseteq X_{0} \times Y$ be $k$-open but not open. Since $U$ is not open in $X \times Y$ it suffices to show that $U_{0}=U \cap\left(X_{0} \times K\right)=U \cap(X \times K)$ is relatively open for each compact subset $K$ of $Y$. But $X_{0} \times K$ is a $k$-space and hence the $k$-open subset $U_{0}$ of $X_{0} \times Y$ is open in $X_{0} \times K$. Since $X_{0} \times K$ is open in $X \times K$, it follows that $U_{0}$ is open in $X \times K$.

2.2 Construction of 1.1. Let $Y$ be a metric space which is not locally compact, and let $X_{0}$ be a $k$-space such that $X_{0} \times Y$ is not a $k$-space [4]. Let $X$ be the one-point compactification of $X_{0}$. Since $X_{0}$ is open in $X$, and since $X_{0} \times K$ is a $k$-space for each compact subset $K$ of $Y$ by a theorem of D. E. Cohen [3], Lemma 2.1 shows that $X \times Y$ is not a $k$-space.

Let us note that the space $X \times Y$ just constructed is not even a quasi- $k$ space: Since each countably compact subset of $Y$ is compact, each countably compact subset $K$ of $X \times Y$ is contained in the compact set $X \times \pi_{Y}(K)$, and hence each $k$-open subset is quasi- $k$-open.

Incidentally, the construction above shows that if $Y$ is a space whose compact subsets are Hausdorff, in particular if $Y$ is Hausdorff or regular, 
then $X \times Y$ is a $k$-space for each $k$-space $X$ if and only if $X \times Y$ is a $k$-space for each compact space $X$. By way of contrast, it is shown in [4] for $Y$ regular that $X \times Y$ is a $k$-space for each $k$-space $X$ if and only if $X \times Y$ is a $k$-space for each paracompact Hausdorff $k$-space $X$ (if and only if $Y$ is locally compact).

\section{Construction of Example 1.2.}

3.1 Lemma. Let $X_{0}$ be an open subspace of a space $X$, and let $Y$ be a space such that, for each countably compact subset $K$ of $Y, X_{0} \times K$ is a quasi-k-space. If $X_{0} \times Y$ is not quasi-k-space, then neither is $X \times Y$.

Proof. Exactly like that of 2.1 .

Let us remark that the hypothesis that $X_{0} \times K$ be a quasi- $k$-space for each countably compact subset $K$ of $Y$ will hold if $Y$ is metric and $X_{0}$ is any quasi- $k$-space, or if $Y$ is regular and $X_{0}$ is a quasi- $k$-space which can be embedded as a subspace of a sequential space [8]. Before constructing our second example, we need some information about spaces which can be embedded as open subspaces of countably compact Hausdorff spaces.

Let us say that a space has the property $\left({ }^{*}\right)$ if for each point $x$ in $X$ and any sequence $\left\{U_{n}\right\}$ of neighborhoods of $x$ there exists a neighborhood $U$ of $x$ such that for each $n$ the set $U \backslash U_{n}$ is contained in some compact subset of $X$.

3.2 Lemma. Let $X$ be a space with the property (*) and let $Y$ be any regular Hausdorff space containing $X$ as a dense subspace. If $\left\{F_{n}\right\}$ is any sequence of closed subsets of $Y$ having $\bigcup_{n} F_{n}$ contained in $Y \backslash X$ then the closure of $\bigcup_{n} F_{n}$ is also contained in $Y \backslash X$.

Proof. Let $F_{n}$ be a sequence of closed subsets of $Y$ which do not meet $X$ and let $x$ be any point of $X$. Choose open neighborhoods $V_{n}$ of $x$ in $Y$ whose closures do not meet $F_{n}$, set $U_{n}=V_{n} \cap X$, and let $U$ be a neighborhood of $x$ in $X$ which has the property that for each $n$ the set $K_{n}=\mathrm{cl}_{X}\left(U \backslash U_{n}\right)$ is compact. Note that $K_{n}=\operatorname{cl}\left(U \backslash U_{n}\right)$ since, being compact, it is closed in the Hausdorff space $Y$. Now choose an open subset $V$ of $Y$ with $V \cap X=U$ and note that $V \subseteq$ cl $U$ since $X$ is dense. Hence

$$
V \subseteq \operatorname{cl} U_{n} \cup \operatorname{cl}\left(U \backslash U_{n}\right) \subseteq V_{n} \cup K_{n} \subseteq Y \backslash F_{n}
$$

for all $n$, so $V$ does not meet $\bigcup_{n} F_{n}$. Hence the closure of $\bigcup_{n} F_{n}$ is contained in $Y \backslash X$.

3.3 Proposition. Of the following conditions, (i) and (ii) are equivalent and are implied by (iii). 
(i) $X$ can be embedded as an open subspace of a countably compact Hausdorff space.

(ii) $X$ can be embedded as a subspace of a countably compact Hausdorff space $Y$ so that $Y \backslash X$ is countably compact.

(iii) $X$ is a completely regular Hausdorff space with the property (*).

Proof. (i) $\Rightarrow$ (ii): Since each closed subspace of a countably compact space is countably compact, this is clear.

(ii) $\Rightarrow$ (i): Suppose $X$ is a subspace of $Y$ where $Y$ and $Y \backslash X$ are countably compact Hausdorff, and let $Z$ be the set $Y$, topologized by calling a subset open if it is either open in $Y$ or relatively open in $X$. Since $X$ is open in $Z$, it suffices to show that $Z$ is countably compact, so let $\mathscr{U}$ be a countable open cover of $Z$. Write $\mathscr{U}$ as $\mathscr{W} \cup\{V \cap X: V \in \mathscr{V}\}$, where $\mathscr{W}$ and $\mathscr{V}$ are countable families of open subsets of $Y$. Now $\mathscr{W}$ covers $Y \backslash X$ and hence contains a finite subcover $\mathscr{U}_{1}$, and $\mathscr{W} \cup \mathscr{V}$ covers $Y$ and hence contains a finite subcover $\mathscr{U}_{2}$. But then $\mathscr{U}_{1} \cup\left\{U \cap X: U \in \mathscr{U}_{2}\right\}$ is a finite refinement of $\mathscr{U}$.

(iii) $\Rightarrow$ (ii): $\beta X \backslash X$ is countably compact. Indeed, by Lemma 3.2 each countable subset of $\beta X \backslash X$ has compact closure.

Notice that the proof of the equivalence of (i) and (ii) in 3.3 can be modified so as to characterize, for example, spaces which can be embedded as open subspaces of Lindelöf Hausdorff spaces.

The class of regular Hausdorff spaces which have the property $\left({ }^{*}\right)$ includes all locally compact spaces and all $P$-spaces, these being in a sense the extremes. The space $X_{0}$ described below also satisfies these hypotheses and is neither locally compact nor a $P$-space.

3.4 Construction of 1.2. Let $N$ denote the positive integers, $I$ the unit interval, and let $X_{0}$ be the quotient of $N \times I$ formed by identifying the set $N \times\{0\}$ to a point, $x_{0}$. We will show that $X_{0}$ has the property $(*)$. That points with compact neighborhoods satisfy this condition is clear, so it suffices to consider a sequence of neighborhoods of $x_{0}$, say $\left\{U_{n}\right\}$, where $U_{n}=\left\{x_{0}\right\} \cup \bigcup_{n}\left(\{m\} \times U_{m n}\right)$ where each $U_{m n}$ is an open interval with left end point zero. Set $U=\left\{x_{0}\right\} \cup \bigcup_{m} \bigcap_{n=m}\left(\{m\} \times U_{n m}\right)$, and notice that for each $n, U \backslash U_{n}$ is contained in $\{1, \cdots, n\} \times I$. Thus $U$ is a neighborhood of $x_{0}$ such that the closure of $U \backslash U_{n}$ is compact for each $n$, as desired.

Now let $Y$ be any metric space which is not locally compact. By [4, Theorem 4.2], $X_{0} \times Y$ is not a $k$-space. Since each countably compact subset of $X_{0} \times Y$ is contained in a compact subset, for example its closure, it follows that $X_{0} \times Y$ is not a quasi- $k$-space. By Proposition 3.3, $X_{0}$ can be embedded as an open subspace of a countably compact Hausdorff space $X$. Finally, by Lemma $3.1, X \times Y$ is not a quasi- $k$-space. 


\section{REFERENCES}

1. A. V. Arhangel'skiī, On a class of spaces containing all metric and all locally bicompact spaces, Dokl. Akad. Nauk. SSSR 151 (1963), 751-754=Soviet Math. Dokl. 4 (1963), 1051-1055. MR 27 \#2959.

2. - Bicompact sets and the topology of spaces, Trudy Moskov Mat. Obšč. 13 (1965), 3-55=Trans. Moscow Math. Soc. 13 (1965), 1-62. MR 33 \#3251.

3. D. E. Cohen, Spaces with weak topology, Quart. J. Math. Oxford Ser. (2) 5 (1954), 77-80. MR 16, 62.

4. E. Michael, Local compactness and Cartesian products of quotient maps and $k$-spaces, Ann. Inst. Fourier (Grenoble) 18 (1968) fasc. 2, 281-286. MR 39 \#6256.

5. - A quintuple quotient quest (to appear).

6. K. Morita, Some properties of M-spaces, Proc. Japan Acad. 43 (1967), 869-872. MR 37 \#3517.

7. J. Nagata, Quotient and bi-quotient spaces of M-spaces, Proc. Japan Acad. 45 (1969), 25-29. MR 39 \#6278.

8. N. Noble, Products of quotient maps and spaces with weak topologies (to appear).

Canary Road, Westlake, Oregon 97493 